\title{
Exploring the Pathological Role of Collagen in Paravertebral Muscle in the Progression of Idiopathic Scoliosis
}

\author{
Haidong Peng, ${ }^{1}$ Feng Jin, ${ }^{2}$ Depeng Meng, ${ }^{3}$ Jun Li, ${ }^{1}$ Shuhan Yu, ${ }^{1}$ Shen Zhang $\mathbb{D},{ }^{1}$ \\ and Guigang Zeng iD 1 \\ ${ }^{1}$ Department of Rehabilitation, Changzheng Hospital, Second Military Medical University, Shanghai, China \\ ${ }^{2}$ Shanghai University of Medicine \& Health Sciences, Shanghai, China \\ ${ }^{3}$ Department of Orthopedics, Changzheng Hospital, Second Military Medical University, Shanghai, China
}

Correspondence should be addressed to Shen Zhang; johnsonzs33@smmu.edu.cn and Guigang Zeng; newbees@smmu.edu.cn

Received 28 February 2020; Revised 20 June 2020; Accepted 14 July 2020; Published 3 August 2020

Academic Editor: Maxim E. Darvin

Copyright (C) 2020 Haidong Peng et al. This is an open access article distributed under the Creative Commons Attribution License, which permits unrestricted use, distribution, and reproduction in any medium, provided the original work is properly cited.

\begin{abstract}
Background. Paravertebral muscle (PVM) is considered as a contributing factor of idiopathic scoliosis (IS); collagen is crucial for maintaining the mechanical properties of PVM, but only a few researches have described this field. In this study, we observed the muscle stiffness of PVM and the curvature of the spine by adjusting the content of collagen in PVM of rats and explored the role of collagen in the progression of IS. Methods. 32 female Sprague Dawley rats were randomly divided into four groups: neutralizing antibody (NA) group (group 1), normal control group (group 2), IS group (group 3), and IS with NA group (group 4). TGF- $\beta 1$ NA was injected into PVM in group 1 and group 4, while Normal saline in group 2 and group 3. The Cobb angle and muscle stiffness were measured before and after injection; the rats were sacrificed at one week after injection, and performed histological, Western Blot, and qRT-PCR examinations. Results. X-rays showed that scoliosis occurred in group 1 and relieved in group 4. The stiffness of PVM was decreased significantly on the convex side in group 1, while on the concave side in group 4 . The expression of TGF- $\beta 1$ and COL1 on the concave side in IS rats (group 3) was significantly increased than that in normal rats (group 2 ), the concentration of COL1 and COL3 in group 3 was significantly higher than that in group 2 , and the addition of TGF- $\beta 1$ NA significantly downregulated COL1 and COL3 in group 1 and group 4 . The concentration of COL1 in convex PVM was negatively related to Cobb angle in group 1 and group 2, and in concave PVM was positively related to Cobb angle in group 3 and group 4 . However, no significant correlation was found between COL3 and Cobb angle in group 3 and group 4. Conclusions. Asymmetric biomechanical characteristics of PVM was an important etiological factor of IS, which was directly correlated with collagen, it could be adjusted by local intramuscular injecting of TGF- $\beta 1 \mathrm{NA}$, and finally had an effect on the shape of the spine.
\end{abstract}

\section{Introduction}

The idiopathic scoliosis (IS) was considered as a multifactorial disease, which was been proposed attributed to a number of factors, such as genetic, biochemical, and hormonal factors [1-4]. In addition, asymmetric loading on the spinal column may play roles in the onset and development of IS. The structures stabilizing the spine, including paravertebral muscle (PVM), have been suggested as implicated in the pathology of IS $[5,6]$.

Mechanical properties of muscle tissue are crucial in the biomechanical balance of the human body [7]. The biomechanical characteristics of PVM has been suggested as being scoliogenic (inducing scoliosis) or counteracting scoliosis in the initial development and maintenance of IS $[8,9]$. Muscle tissue is a combination of muscle fibers and collagen fibers, muscle fibers are surrounded by connective tissue made of collagen fibers [10]. The active behavior of muscle usually refers to the contractile response of muscular fibers. Meanwhile, mainly responsible for the passive behavior of muscle is determined by collagen fibers [11, 12]. Previous studies have shown abnormal paraspinal electromyographic activity in PVM of IS patients $[13,14]$, which has been proved to be connected with the neuromuscular disorder [15]. However, the passive biomechanical characteristics related to collagen fibers in PVM are still under further research. 


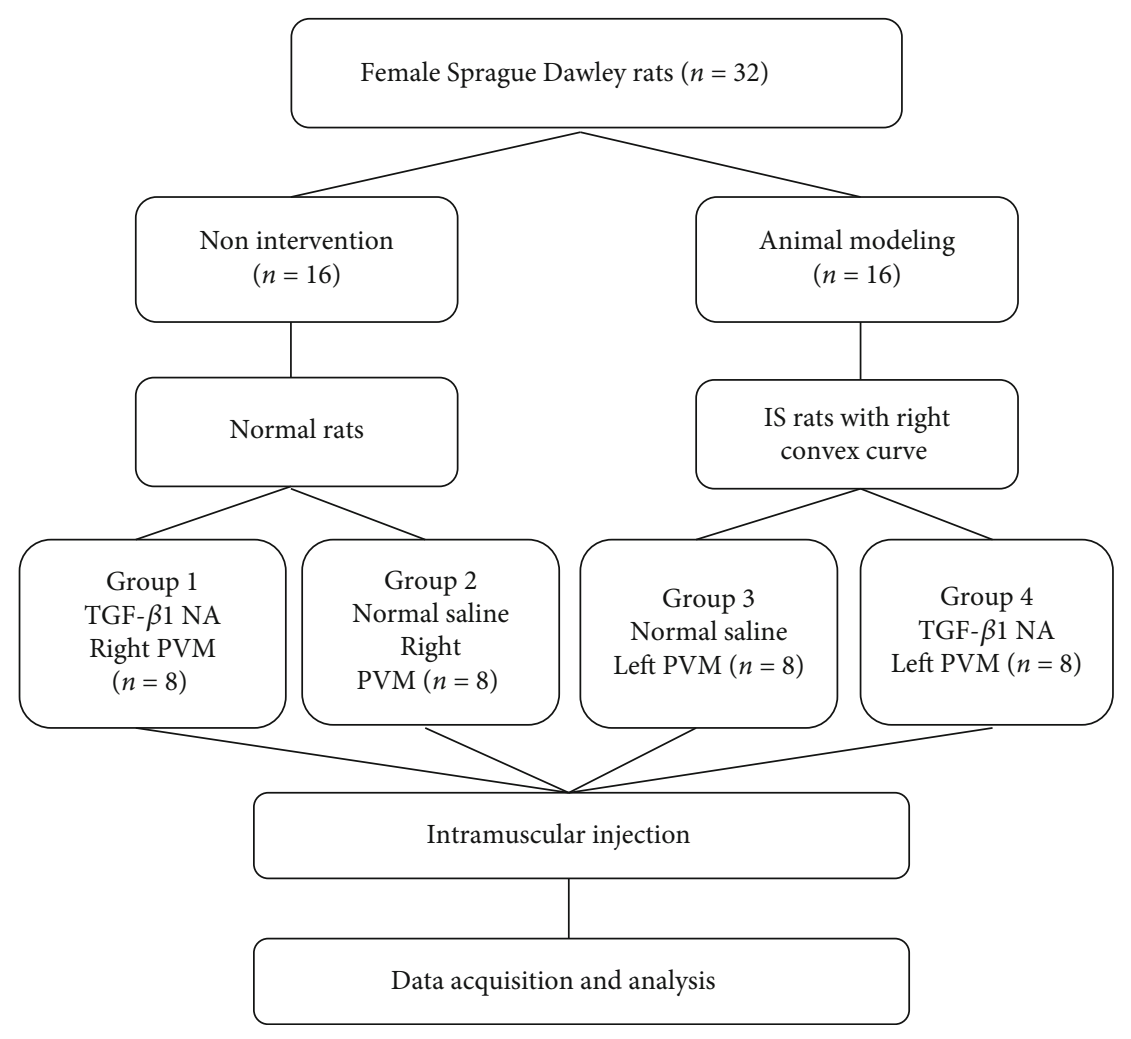

Figure 1: The study design.

Despite the comparatively small amount of collagen, which accounts for only $1-10 \%$ of the muscle dry weight [16], it acts a central role in force transmission as it ensures the integrity and proper functioning of the entire muscle [17]. Fibrosis in the concave PVM was one of the noticeable pathological changes in IS patients [18]; It indicates that excessive collagen in concave PVM was an interesting target. We hypothesized that different collagen content in PVM resulted in asymmetry tension, and possibly related to the occurrence of IS. Transforming growth factor- $\beta 1$ (TGF- $\beta 1$ ) is the most important cytokine regulating collagen $[19,20]$; in this study, TGF- $\beta 1$ neutralizing antibody (NA) were injected into the right PVM of normal rats (to reduce collagen in right PVM) and left PVM of IS rats with right convex curve (to reduce the collagen in left PVM with collagen fibers hyperplasia). This could clarify whether the collagen in PVM would in fact induce the spinal deformity by asymmetric tension, thus having a scoliogenic effect, where changes of the spinal curve after reduction of collagen would happen.

\section{Materials and Methods}

2.1. Animals. 32 3-month-old virgin female Sprague Dawley rats weighing 200-220g were obtained from Shanghai Lab Animal Research Center. All experimental procedures conducted were approved by the Institutional animal care and use committee at the university of the second military medical university, Shanghai, China. In this study, rats were randomly divided into 4 groups of 8 animals each: TGF- $\beta 1$ NA group (group 1), normal control group (group 2), IS group (group 3), and IS with TGF- $\beta 1$ NA group (group 4), Figure 1. All groups were maintained on a laboratory rodent chow diet and housed in a laboratory at $22^{\circ} \mathrm{C}$, humidity $40 \%$, and light controlled (under a $12 \mathrm{~h}$ light and $12 \mathrm{~h}$ dark cycle).

2.2. Modeling Methods. Group 1: anesthesia was maintained by isoflurane inhalation, 10 points were evenly marked on the line connecting right scapula to the ipsilateral iliac wing ( $5 \mathrm{~mm}$ beside the spinous process) as the intramuscular injection points of PVM in a prone position, Figure 2(a). Each point was injected with $0.1 \mathrm{ml}$ TGF- $\beta 1 \mathrm{NA}$ (Mouse monoclonal antibody against rat TGF- $\beta 1$; ab64715, Abcam, $0.5 \mathrm{mg} / \mathrm{ml})$ diluted with normal saline $(2.0 \mu \mathrm{g} / \mathrm{ml})$, once a week, three times in total.

Group 2: normal saline was injected into the right PVM. The injection site, dose, and frequency were the same as that of group 1, Figure 2(a).

Group 3: rats were anesthetized intraperitoneally with $2 \%$ barbitalum natricum $(40 \mathrm{mg} / \mathrm{kg})$ and underwent subcutaneous left scapula-to-ipsilateral iliac wing tethering procedure with nonabsorbable suture. The lower angle of the scapula and iliac wing were drawn together to $90 \%$ of the original distance, which made the spine convex toward the right side. Amputating the forelimbs at a high humeral level and the tails at the root to create the bipedal rats. After removing the forelimbs and tail, the bipedal rats were housed in special high cages with raised food and water to ensure that they maintained a standing posture most of the time; both food and water were gradually elevated in the cage as the bipedal rats grew $[21,22]$. The sutures were cut to complete the 


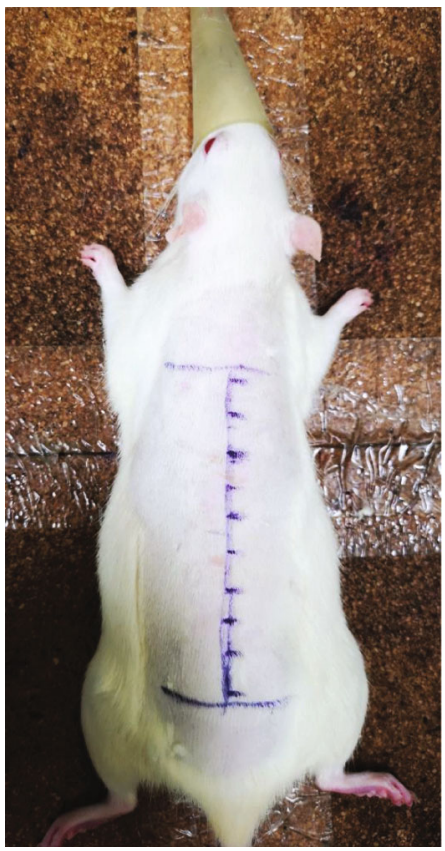

(a)

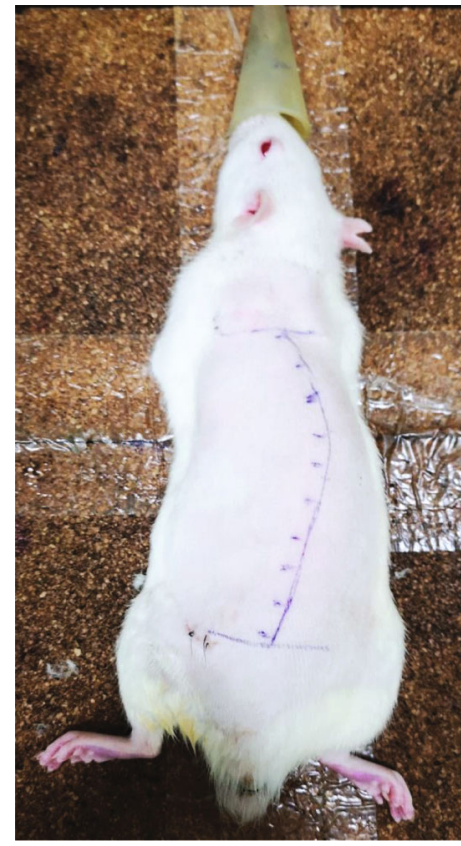

(b)

FIGURE 2: (a) Intramuscular injection points of PVM in group 1 and group 2. (b) Intramuscular injection points of PVM in group 3 and group 4.

modeling at postoperative 8 weeks. One week after the completion of modeling, the Cobb angle was greater than $10^{\circ}$, indicating that the modeling was successful. Normal saline was injected into the left PVM; the injection site, dose, and frequency were the same as that of group 1, Figure 2(b).

Group 4: one week after IS models were made successfully, TGF- $\beta 1$ NA was injected into the PVM of the left side. The injection site, dose, and frequency were the same as that of group 3, Figure 2(b).

To avoid the errors by employing different operators, all experimental procedures were accomplished by the same experimenter and assistant.

2.3. Imaging Examination. X-ray of full-length radiographs was taken by In-Vivo Imaging System FX Pro (Kodak, USA) to evaluate the Cobb angle before injection and one week after injection. Rats in all groups were anesthetized by isoflurane and involved positioning them as straight as possible without any applied traction. Cobb angle at the primary curve in the coronal planes were measured by Digimizer software. The location of the upper or lower vertebra was together identified by two experienced evaluators of radiology. If there were different views, the average was selected.

2.4. Muscle Stiffness. Muscle stiffness is defined as the degree of deformity of the muscle to given a pressure [23, 24]. Applying the same pressure, the higher degree of muscle stiffness is, the smaller is the tissue displacement. It has been proved to be a reliable noninvasive examination by many researches that the muscle stiffness can be measured by the force-displacement curve [25] In this study, M_tone Soft Tissue Elasticity Meter (JZL-111, Tianjin Mingtong Century Technology Co., Ltd.) was applied to examine the muscle stiffness of bilateral PVM after radiological examination.
The M_tone Soft Tissue Elasticity Meter consists of a metal probe, surrounded by a metal shaft. When the tester applies vertical downward compressive force, the shaft remains stationary on the surface of the skin while the probe compresses the tissue to produce deformation, Figure 3(a). In the process of detection, all data were registered and stored in a computer attached to the device, and two real-time force-displacement curves, loading curve, and unloading curve showed on computer based on these data simultaneously. In this study, muscle stiffness of PVM was reflected by the displacement at $200 \mathrm{~g}$ pressure $\left(\mathrm{D}_{200}\right)$ on the loading curve. The proportion of "banana" area (PBA) between the two curves in the total area under the loading curve, which is correlated with the degree of muscle spasm or tissue stiffness, the higher PBA was, the less muscle spasm it was [26], Figure 3(b).

During the measurement, rats were anesthetized by inhalation of isoflurane with lying prone; reference test points were marked on both sides at the apex of curve $5 \mathrm{~mm}$ beside the spinous process, Figure 3(c).

2.5. Tissue Specimens. PVM was harvested from the right side (groups 1 and 2) or left side (groups 3 and 4). Muscle samples were placed in separate sterile tubes and immediately stored in liquid nitrogen. Frozen samples were stored at $-80^{\circ} \mathrm{C}$ until analysis.

2.6. Histological Evaluation of Fibrosis. The muscle samples were fixed in $10 \%$ formalin, embedded in paraffin blocks, and cut into $5 \mu \mathrm{m}$ thickness slices. Masson trichrome staining was carried out to estimate the severity of fibrosis in PVM as follows [27]: Cross sections were deparaffinized, rehydrated, washed in running water for $2 \mathrm{~min}$, immersed in Weigert's iron hematoxylin for $15 \mathrm{~min}$, and rinsed in distilled water, then washed in running water for $5 \mathrm{~min}$. Then, sections were 


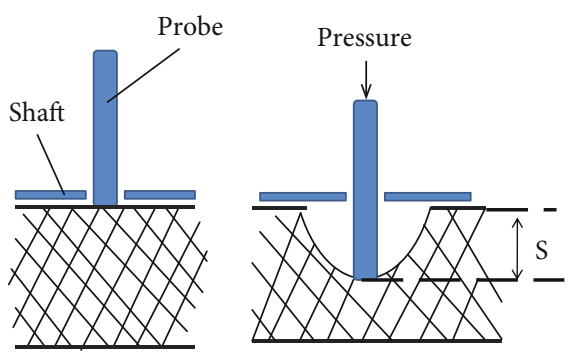

(a)

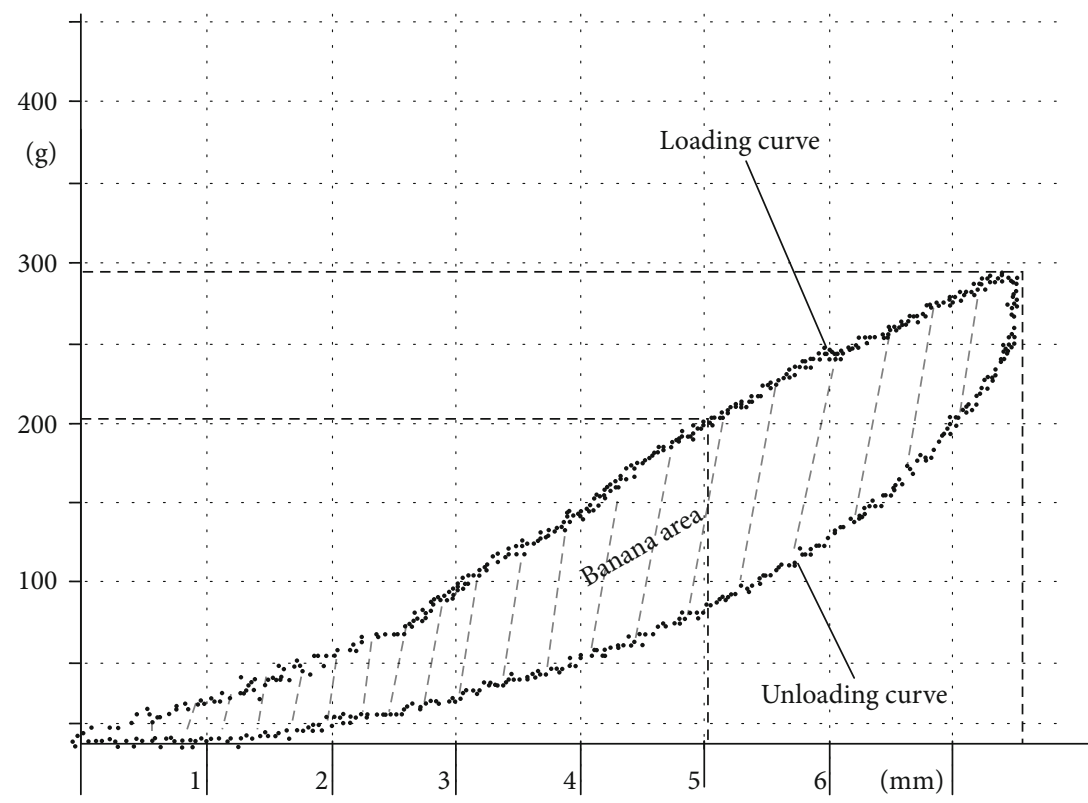

(b)

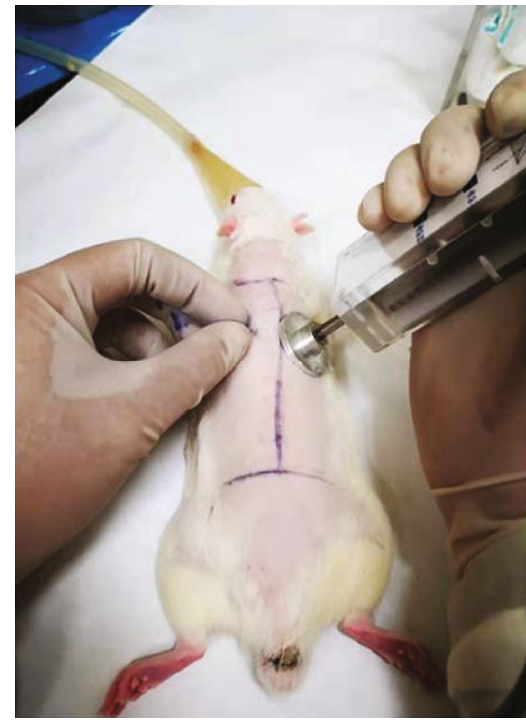

(c)

Figure 3: (a) Schematic diagram of muscle stiffness test principle. (b) The interface of data acquisition and data displaying, $x$-axis shows the distance travelled, whereas the $y$-axis to show the vertical downward compressive force in each position. (c) The operation method of M_tone Soft Tissue Elasticity Meter.

stained with $1 \%$ Biebrich scarlet-acid fuschin for $15 \mathrm{~min}$ and washed in distilled water for $5 \mathrm{~min}$. After differentiation in 2.5\% phosphomolybdic-phosphotungstic acid solution for $15 \mathrm{~min}$, sections were transferred directly into $2.5 \%$ aniline blue solution for $12 \mathrm{~min}$. Muscle sections were then differentiated in $1 \%$ acetic acid solution for $3 \mathrm{~min}$, dehydrated in $95 \%$ and $100 \%$ ethanol, then cleared in xylene. Muscle fibers were stained red and collagen fibers blue in Masson staining. The ratio of the fibrotic area to the total cross-sectional area was calculated to estimate the severity of fibrosis in PVM.

2.7. Western Blot. The protein samples were carefully separated and washed with sterile phosphate-buffered saline. Then, samples were stored in a saline-soaked gauze at $-20^{\circ} \mathrm{C}$ until analysis. Separated proteins were blot transferred onto a nitrocellulose membrane. After blocking with $0.1 \%$ Tween 20 and 5\% nonfat milk in tris-buffered saline at room temperature for $1 \mathrm{~h}$, the membrane was incubated overnight at $4^{\circ} \mathrm{C}$ in the following primary antibody: Anti-TGF beta 1 (Abcam,ab92486), Anti-Collagen I Rabbit pAb (GB110222), Anti-Collagen III Rabbit pAb (GB11023), and $\beta$-actin (Santa Cruz, CA, USA) as an internal control. The intensity
TABle 1: Primers sequences for qRT-PCR.

\begin{tabular}{llc}
\hline Gens & Direction & Primers $\left(5^{\prime}-3^{\prime}\right)$ \\
\hline \multirow{2}{*}{ Gapdh } & Forward & TCTCTGCTCCTCCCTGTTC \\
& Reverse & ACACCGACCTTCACCATCT \\
\multirow{2}{*}{ COL1 } & Forward & GGTTGCAGCCTTGGTTAG \\
& Reverse & TGAGCCAGCAGATTGAGAA \\
& Forward & GGTTTGGAGAATCTATGAATGGTGG \\
& Reverse & GCTGGAAAGAAGTCTGAGGAAGG \\
\hline
\end{tabular}

of protein bands was measured, and the protein expression levels were normalized to $\beta$-actin concentration.

2.8. $q R T-P C R$. To assess the concentration of COL1 and COL3, the muscles were homogenized in Trizol reagent using TissueLyser (TissueLyser II, QIAGEN Inc., Germany). RNA was isolated according to the manufacturer's guidelines. The RNA was reverse transcribed to CDNA and diluted 1:10. Quantitative Real-Time Polymerase Chain Reaction (qRT-PCR) analysis of COL1 and COL3 using the TaqMan ${ }^{\mathrm{TM}}$ Gene Expression Assays (Thermo Fisher Scientific, Canada) Table 1. 

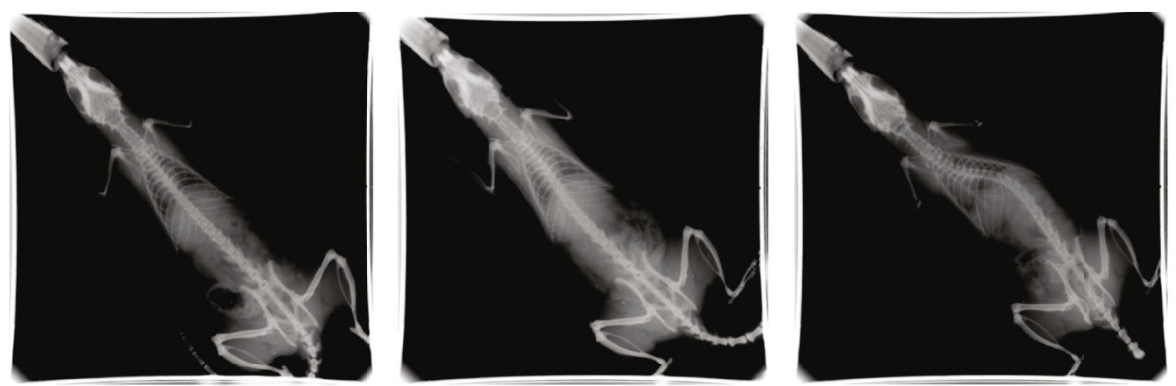

(a) Radiograph of full spine before injection

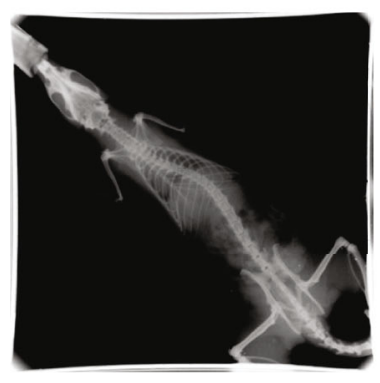

Group 1
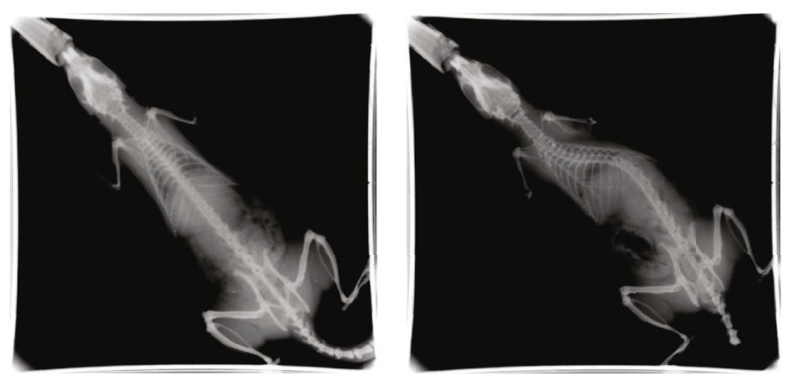

(b) Radiograph of full spine at one week after injection Group 2

Group 3
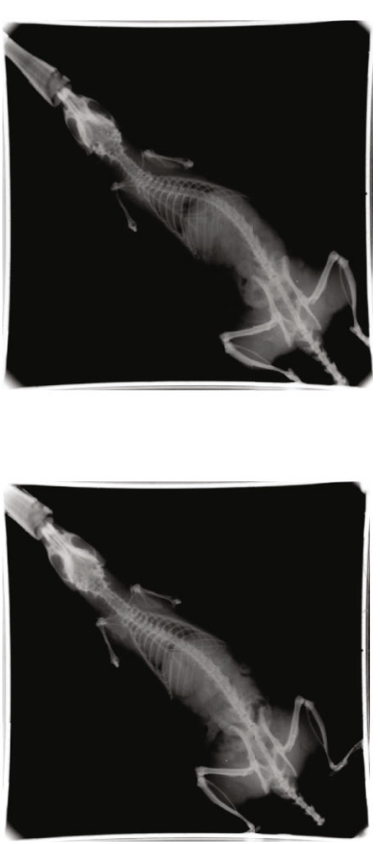

Group 4

Figure 4: Comparison of radiograph of full spine between groups.

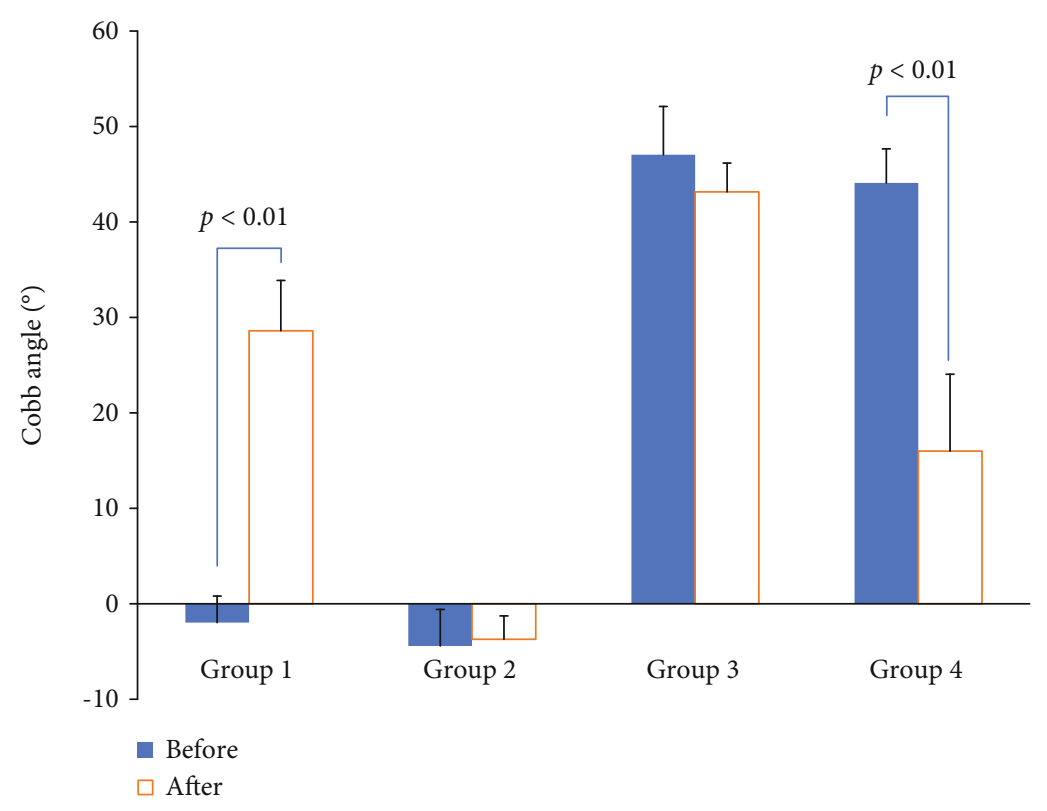

Figure 5: The Cobb angle between groups.

The relative amount of mRNA in the muscle compared with the Gapdh genes was computed according to the comparative $\mathrm{Ct}$ method. We computed the relative gene expression for each of the genes in tissue from the instrumented level by subtracting the $\mathrm{Ct}$ value of an endogenous control gene from the $\mathrm{Ct}$ value of the experimental gene, yielding the $\triangle \mathrm{Ct}$ value. Then, we further normalized $\triangle \mathrm{Ct}$ values of the instrumented samples to the $\triangle \mathrm{Ct}$ values of the internal control discs, yielding the $\triangle \triangle \mathrm{Ct}$ value. Finally, assuming that each strand of cDNA in the sample is copied exactly once per PCR cycle (giving an exponential relation between the cycle number and quantity of cDNA), we used the comparative $\mathrm{Ct}$ value method $\left(2^{-\triangle \triangle \mathrm{Ct}}\right)$ in statistical analyses.

2.9. Statistical Analysis. All statistical analyses were performed using the SPSS 18.0 (IBM Corp, Armonk, NY). Paired $T$ test was used to compare the difference of muscle 


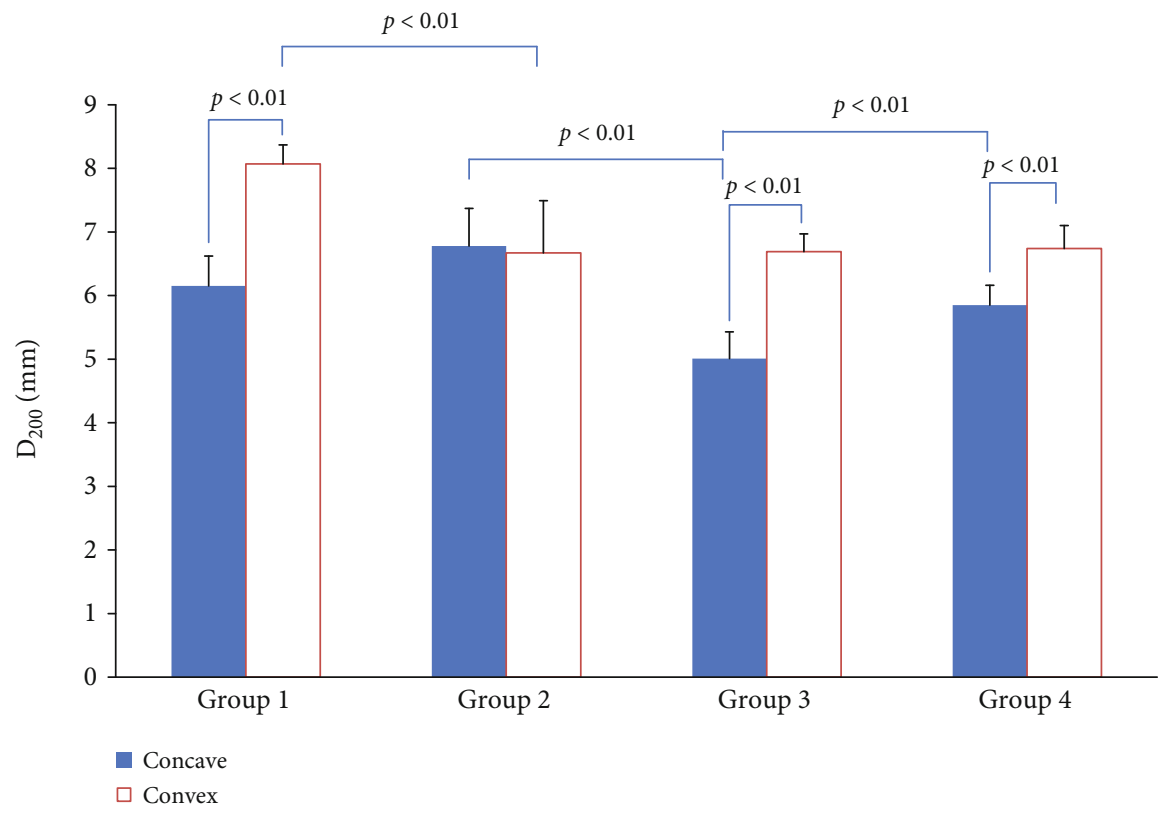

(a)

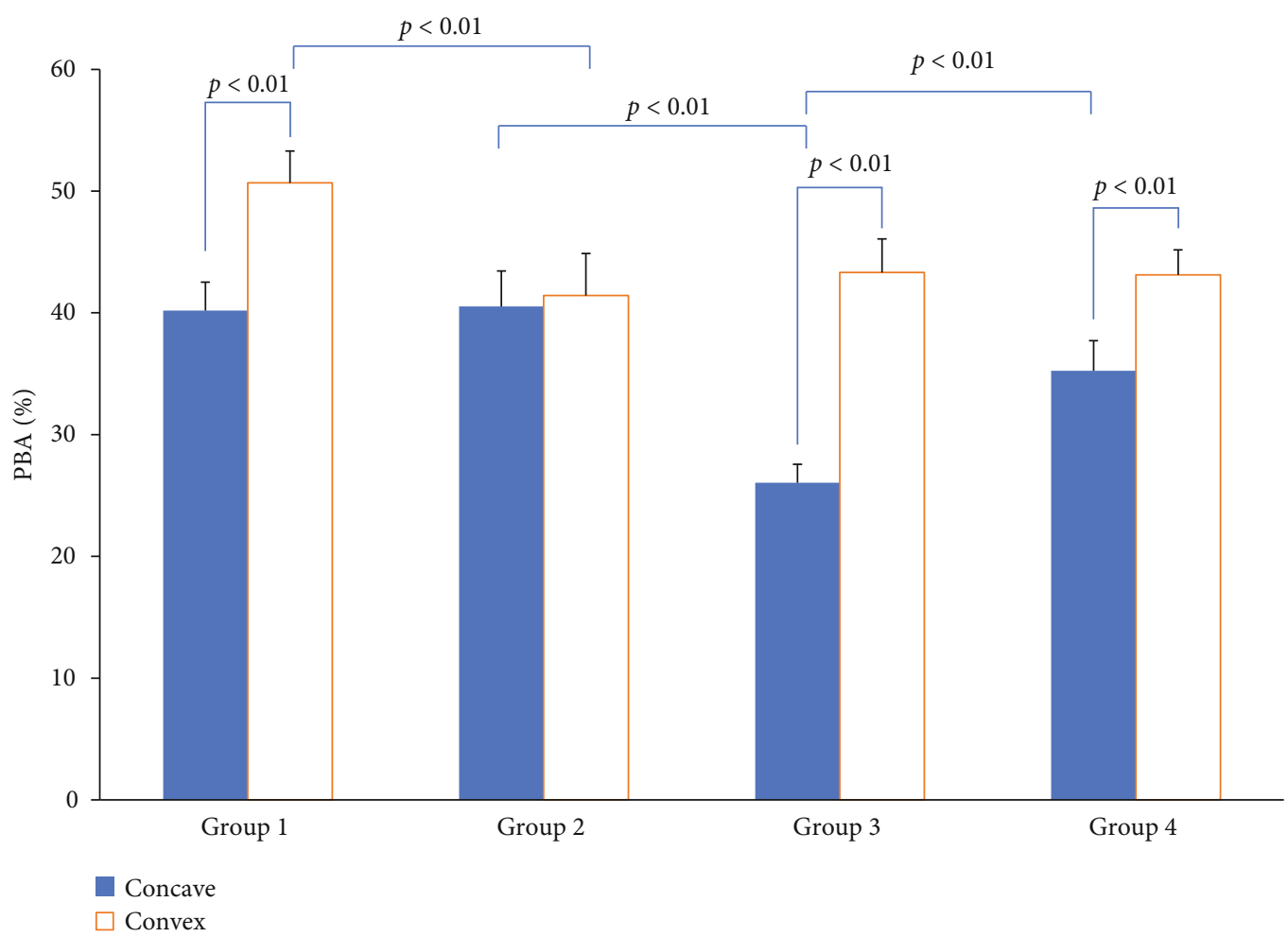

(b)

FIgURE 6: (a) Comparison of bilateral $\mathrm{D}_{200}$ between groups. (b) Comparison of bilateral PBA between groups.

stiffness in bilateral PVM. Independent-Sample $T$ test was used to compare the Cobb angle, protein expression, mRNA concentration, and fibrotic area between groups. Finally, Pearson correlation analysis was conducted to analyze the correlations between parameters. All analyses values were expressed as mean \pm standard deviation (SD), and the significance level of the tests was set at 0.05 .

\section{Results}

3.1. Imaging Examination. One week after injection, $\mathrm{X}$-rays showed that scoliosis occurred in group 1, conversely, relieved in group 4. The Cobb angle in group 1 (ranged from $23.6^{\circ}$ to $42.3^{\circ}$ ) was gradually increased compared with that in group 2 (ranged from $-6.1^{\circ}$ to $6.3^{\circ}$ ). In group 4 , the Cobb 

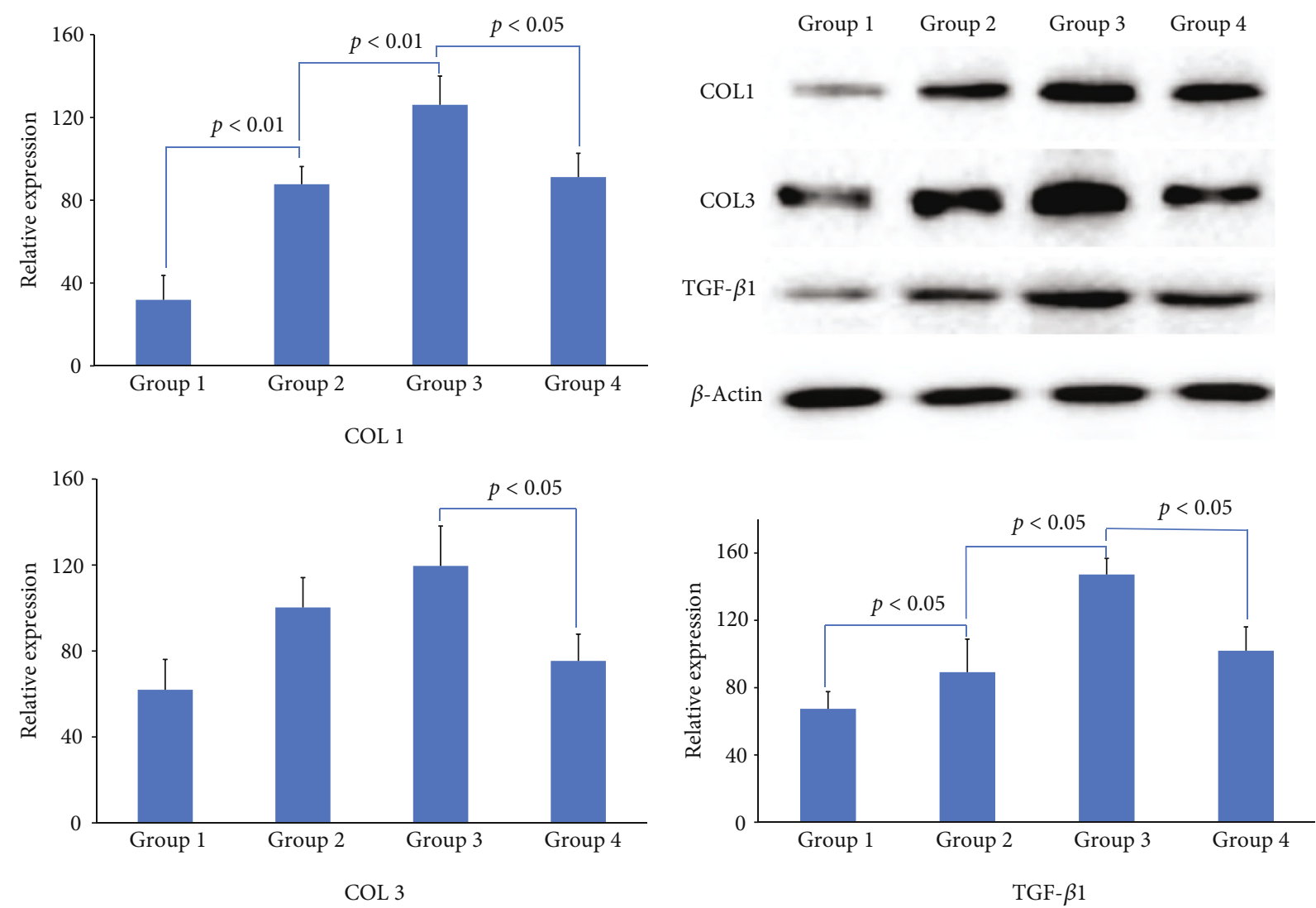

FIGURE 7: Quantification of COL1, COL3, and TGF- $\beta 1$ expression in PVM between groups.

angle (ranged from $8.4^{\circ}$ to $29.5^{\circ}$ ) was decreased significantly than that in group 3 (ranged from $41.7^{\circ}$ to $54.7^{\circ}$ ). Anteroposterior views of spinal radiographs are shown in Figure 4, and overall scoliosis rates recorded are shown in Figure 5.

3.2. Muscle Stiffness. The imbalance muscle stiffness of bilateral PVM widely existed in group 1, group 3, and group 4, in which there were different degrees of scoliotic curve. The stiffness and spasticity of PVM on the concave side in IS rats (group 3) was significantly different from that of normal rats (group 2). After injection, the $\mathrm{D}_{200}$ and PBA was increased significantly on the convex side in group 1 , while was decreased significantly on the concave side in group 4 . Figures 6(a) and 6(b) list the results of the M_tone examination in the bilateral comparison of the scoliosis curve.

3.3. Western Blot. The protein expression of TGF- $\beta 1$ and COL1 on the concave side in IS rats (group 3) was significantly increased than that in normal rats (group $2, p<0.01$ ), COL3 in IS rats also increased, but there was no statistical significance ( $p=0.1332$ ). Addition of TGF- $\beta 1$ NA resulted in a significant decrease of TGF- $\beta 1$ and COL1 in group 1 (vs. group $2, p<0.05$ ) and group 4 (vs. group $3, p<0.05$ ), COL3 decreased in group 1 , but there was no statistical significance (vs. group $2, p=0.3360$ ), Figure 7 .

3.4. qRT-PCR. The concentration of COL1 and COL3 in group 3 was significantly higher than that in group 2 $(p<0.01)$. The addition of TGF- $\beta 1$ NA significantly down- regulated the concentration of COL1 in group 1 and group $4(p<0.01)$, but the decrease of COL3 in group 4 has no statistical significance $(p=0.1345)$, Figures $8(a)$ and $8(b)$.

3.5. Fibrotic Area Assessment. The proportion of the fibrotic area in concave PVM in group 3 was significantly higher than group $2(p<0.01)$. The positive fibrotic areas were significantly less prevalent in the TGF- $\beta 1$ NA groups (group 1 vs. group 2, $p<0.01$; group 3 vs. group $4, p<0.01$ ), Figures 9(a) and $9(b)$.

3.6. Correlational Analyses. The muscle stiffness of PVM was closely related to the Cobb angle; there was a significant negative correlation between the difference value of muscle stiffness on bilateral PVM $\left(\triangle D_{200}\right.$ or $\left.\triangle \mathrm{PBA}\right)$ and Cobb angle. The concentration of COL1 in convex PVM was negatively related to Cobb angle in group 1 and group 2 and was positively related to Cobb angle in group 3 and group 4 in concave PVM. However, no significant correlation was found between COL3 and Cobb angle in group 3 and group 4, Table 2.

\section{Discussion}

4.1. PVM and IS. The etiology of IS is linked to many factors; studies support that spinal deformity may be associated with PVM atrophy. PVM lesion is more often observed on the concave side among patients with IS, which is positively connected with the progression of IS [6]. The muscle thickness 


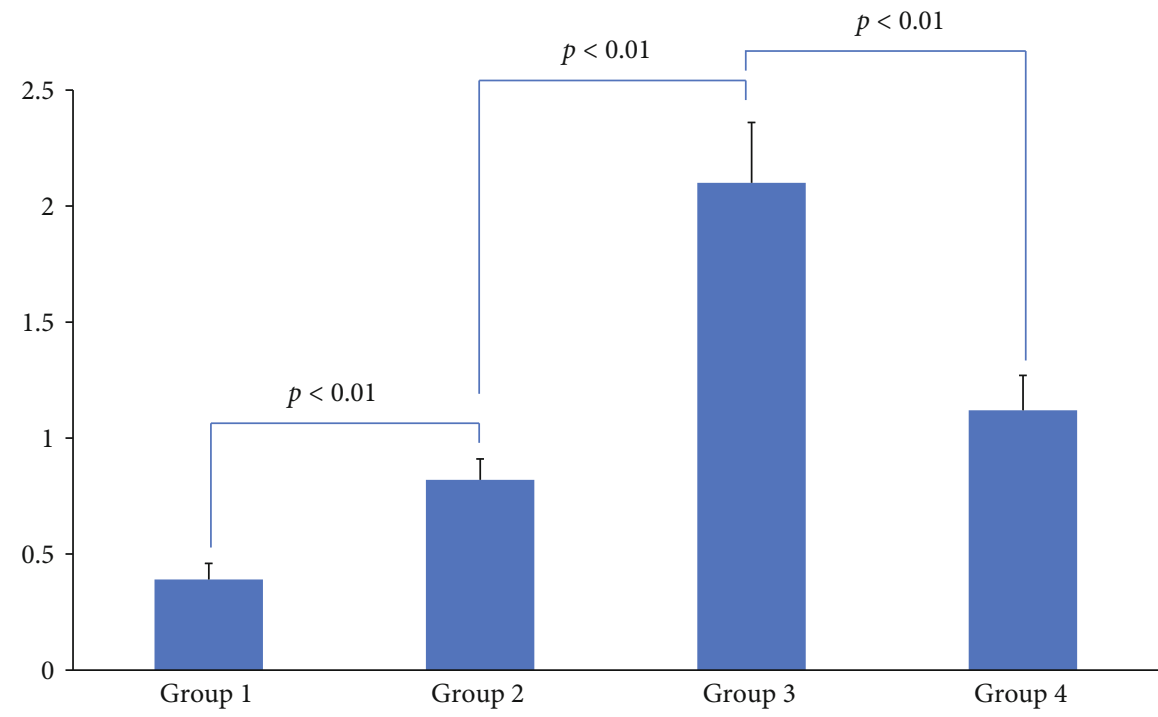

COL 1

(a)

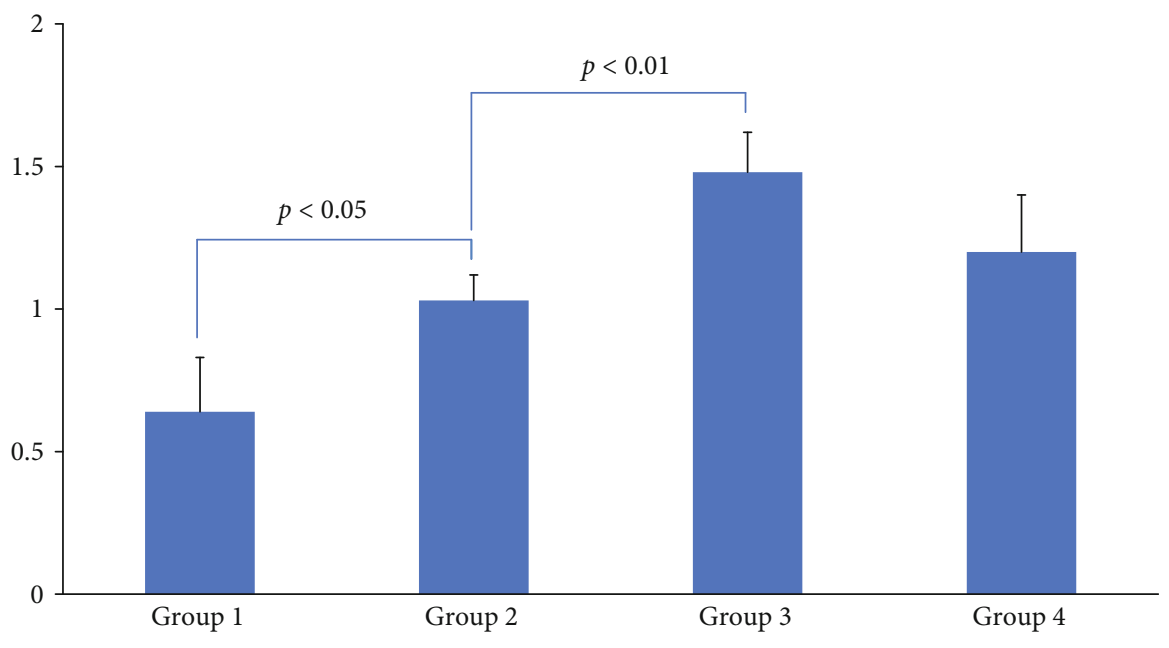

COL 3

(b)

Figure 8: (a) The concentration COL1 in PVM. (b) The concentration COL3 in PVM.

was significantly greater on the concave side of the curve compared with the control's dominant side [28]. The muscle volume and fatty infiltration in deep PVM of adolescent IS patients were imbalance [29]. The type sizes and distributions of muscle fiber were different in the concave and convex sides in IS [30]. Magnetic resonance imaging and histology showed that muscle degeneration was more common on the concave side in patients with IS [31]. The presence of PVM disorder has been suggested as a plausible cause for scoliosis [32].

PVM is vital for the stability and functional movement of the spinal column; tension imbalance is a key factor in the initiating or maintaining scoliotic curvature by muscle contraction [33]. The stiffness of concave PVM was greater than that on the convex side in IS patients. The asymmetric biomechanical characteristics of PVM are related to the severity of scoliosis [8]. We found that the asymmetric muscle stiffness of PVM was well correlated with the Cobb angle that implied PVM played a scoliogenic role by performing a lateral pull or contraction. As we supposed earlier, if the excessive muscle pull by contraction was reduced in the concave side with the subsequent effect in the reduction of Cobb angle in IS rats. Conversely, the unilateral insufficient muscle traction leads to increased Cobb angle, thus creating a scoliosis in normal rats.

4.2. Collagen and the Mechanical Properties of PVM. Mechanical properties of skeletal muscle are crucial in spinal stability, which is determined by its composition. Skeletal muscle is a combination of muscle fibers and connective tissue including collagen fibers [7]. Collagen fibers maintain the integrity of skeletal muscle by binding muscle fibers together 


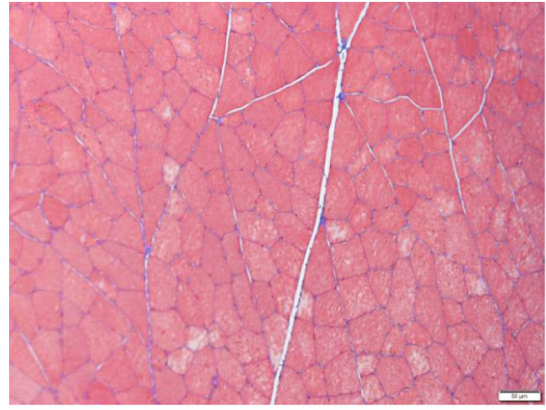

Group 1

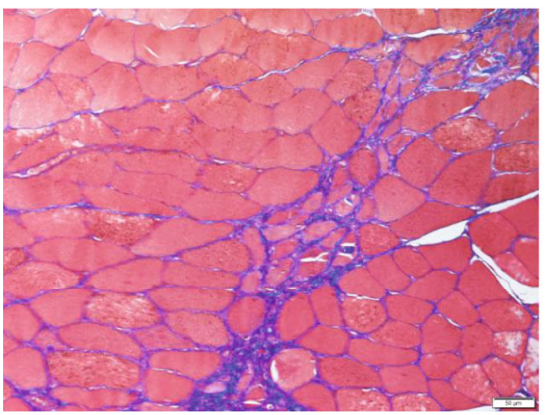

Group 3

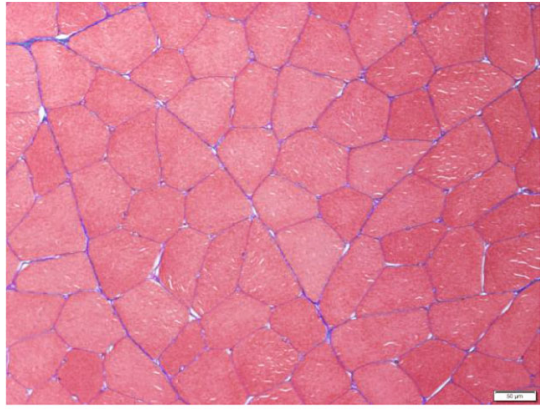

Group 2

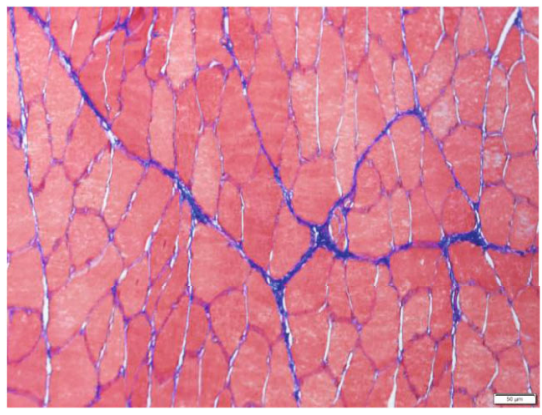

Group 4

(a)

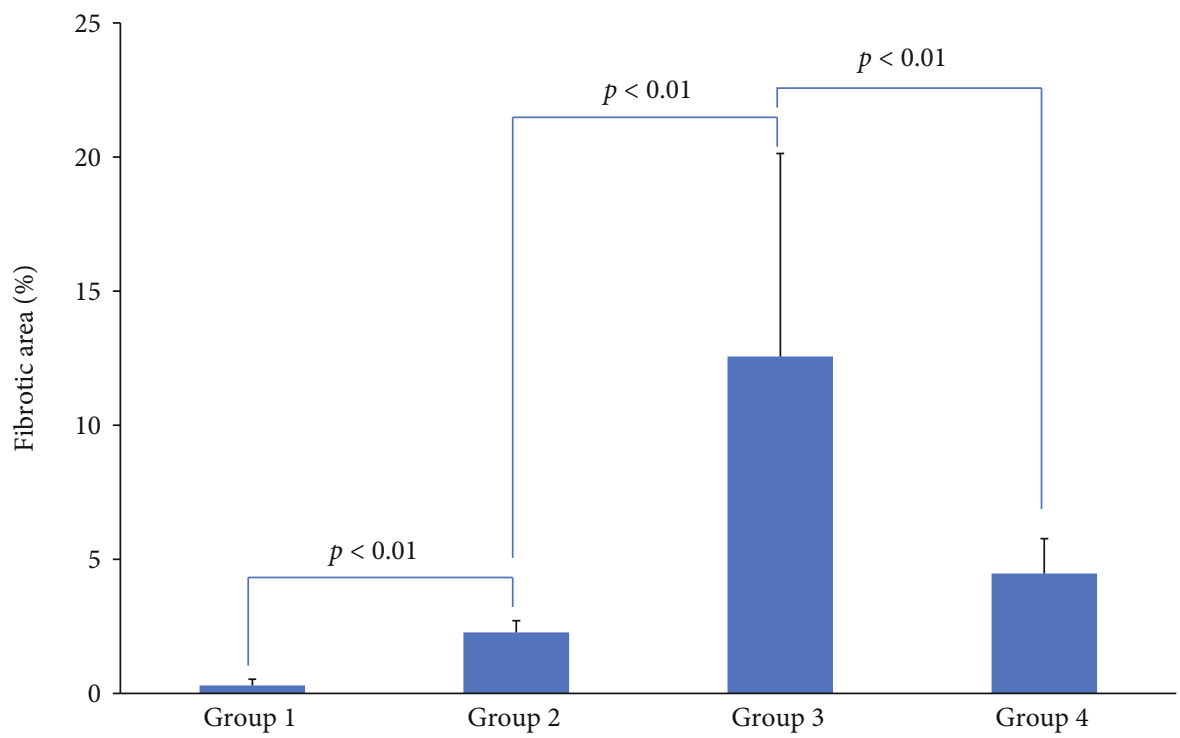

(b)

FIGURE 9: (a) Muscle fibrosis in PVM in different groups. Masson trichrome staining was used to determine the total amount of collagen in the muscle tissue. Collagen was stained blue; myofibers were stained red (Masson staining, $\times 200$, Scale bar on pictures is $50 \mu \mathrm{m}$ ). (b) Quantification of the proportion of fibrotic area to the total cross-sectional area.

and allow the transmission of muscular forces [34]. Skeletal muscle was considered as a three-dimensional network structure of muscle fibers, collagen fibers embedded in an isotropic matrix [35]. From the internal to the external level, the muscle fibers are organized histologically by the surrounding connective tissues of three different properties made of collagen fibers: the endomysium surrounds individual fibers; the perimysium binds muscle fibers into fascicles and the epimysium surrounds the whole muscle $[36,37]$.
The active response of skeletal muscle is responsible for the contractile behavior, usually refers to the response of the muscular fiber $[11,12]$. Meanwhile, the passive mechanical properties of skeletal muscle clearly depend on the size, orientation, and organizational arrangement of collagen fibers, particularly in the perimysium [38]. These pathologic changes of collagen hyperplasia induce muscle contracture and finally increased passive stiffness $[39,40]$. Muscle passive stiffness can contribute to the loss of range of motion about 
TABLE 2: Linear regression analysis of muscle stiffness, collagen with Cobb angle.

\begin{tabular}{|c|c|c|c|c|c|}
\hline & Group & $n$ & Coefficient* & $\begin{array}{c}R^{2} \\
\text { linear }\end{array}$ & $p$ value \\
\hline \multicolumn{6}{|c|}{ Muscle stiffness } \\
\hline$\triangle \mathrm{D}_{200}$ & $\begin{array}{c}\text { Groups } 1 \text {, } \\
2,3,4\end{array}$ & 32 & -0.841 & 0.708 & $<0.001$ \\
\hline$\triangle \mathrm{PBA}$ & $\begin{array}{c}\text { Groups } 1 \\
2,3,4\end{array}$ & 32 & -0.915 & 0.839 & $<0.001$ \\
\hline \multicolumn{6}{|c|}{ Collagen content } \\
\hline \multirow{2}{*}{ COL1 } & Groups 1, 2 & 16 & -0.900 & 0.810 & $<0.001$ \\
\hline & Groups 3, 4 & 16 & 0.923 & 0.852 & $<0.001$ \\
\hline \multirow{2}{*}{ COL3 } & Groups 1,2 & 16 & -0.539 & 0.291 & 0.031 \\
\hline & Groups 3, 4 & 16 & 0.448 & 0.201 & 0.082 \\
\hline
\end{tabular}

*Pearson correlation coefficient.

the spine, leading to further loss of function. Muscle stiffness is directly correlated with total collagen content [41, 42], which is consistent in our experimental results. Furthermore, we found that the muscle stiffness of PVM was positively related to COL1, however, was not correlated with COL3. This discrepancy could be explained by the proportion of different collagen types. In skeletal muscle, COL1 is predominant, whereas COL3 accounts approximately for $10 \%$ of total collagen [43]. COL1 resists tension and COL3 provides structural stability [44], difference of arrangement, and size of collagen fibers resulted in COL1 is more resistant than COL3 [45]. In our study, muscle stiffness and Cobb angle were positively related to COL1 in PVM, while were not correlated with COL3 in group 3 and group 4 . This result suggests that the abnormality of COL1 has a closer relation to the progression of IS.

4.3. Fibrosis of PVM and IS. PVM degeneration presents very frequently on the concave side of the scoliotic curve, which was morphologically abnormal in patients with IS [6]. Fibrosis, an excessive accumulation of collagen, in skeletal muscle impairs its function [40,46], is a cause of increased stiffness of skeletal muscle. TGF- $\beta 1$ is considered critical regulators of physiological fibrogenesis and pathological fibrosis in kidneys, liver, lungs, and skeletal muscles [47]. In previous studies, tissue samples collected from IS patients indicated that TGF- $\beta 1$ in PVM, vertebral cartilaginous endplates, or articular cartilages of the scoliosis apex was significantly higher on the concavity [48]; the fibrosis of muscular samples was more serious on the concave side [18]. With the deteriorated muscular fibrosis on the concavity, the prominent difference of TGF- $\beta 1$ was observed on both sides showed that TGF- $\beta 1$ was involved either as an etiological factor or a secondary change in the scoliosis progression [49].

To our knowledge, this was the first study, in which PVM was influenced directly by intramuscular injection of TGF- $\beta 1$ $\mathrm{NA}$ in order to explore the role of collagen in the progression of IS. Collagen in our hypothesis was being an important step for the further exploration and understanding of the etiology of IS. TGF- $\beta 1$ NA were injected into the right PVM of normal rats to reduce the content of collagen, resulting in a drop of muscle stiffness on the right side, and produce a right convex curve. Similarly, the injection of TGF- $\beta 1$ NA into the concavity of IS rat could alleviate the degree of PVM stiffness and reduce the Cobb angle. We found that the content of collagen was closely related to muscle stiffness. By reducing the content of collagen, we could adjust the asymmetric biomechanical characteristics of bilateral PVM and finally had an influence on the formation of the spinal column.

\section{Conclusions}

In conclusion, asymmetric biomechanical characteristics of PVM is an important etiological factor of IS, which is directly correlated with collagen. By local intramuscular injecting of TGF- $\beta 1$ NA to reduce the content of collagen in PVM, the asymmetric tension of PVM can be adjusted; finally, it has an effect on the shape of the spine. The identification of significant asymmetry in the content of collagen between the concave and convex side of the scoliotic curve could suggest a new perspective of progression risk and aid in developing novel therapeutics to combat structural changes of the spine.

\section{Limitations}

However, in this study, we detect an antifibrosis treatment for short-term effects and only focused on the quantity of collagen. It is obvious that in such multiple factorial disease as IS, one factor cannot be the only participant responsible for spine curvature dysfunction, precise mechanisms of collagen still remain elusive. Thus, further tests are necessary to determine the ratio of COL1/COL3, which is important and might influence the biomechanical property of PVM, or the doseeffect relationship of TGF- $\beta 1$ NA. These further studies will bring us additional to understand the roles of collagen in the progression of scoliosis that may allow us to effectively improve spinal deformity.

In addition, in groups 3 and 4, we adopted the approach of bipedal unilateral tethering to establish scoliosis model, which was a mature and standard modeling method widely used in studies of scoliosis, while adopted quadrupedal rats in groups 1 and 2. Due to rodents exhibit a preference for erect walking and phylogenetically, they are still quadrupeds, some studies suggested that the upright posture may not be the difference of some previously reported between bipedal and quadrupedal rats, bipedal rat spends no more time in an upright position compared with quadrupedal rats [50, 51]. The gravity seemed to have been a relatively minor factor and would not lead to significant error of the experimental results. However, the consistency between groups still need improvement in our following study, and how gravity and collagen contribute to scoliosis deserves further study.

\section{Abbreviations}

IS: Idiopathic scoliosis

PVM: Paravertebral muscles

TGF: Transforming growth factor

NA: Neutralizing antibody

COL: Collagen 
$\mathrm{D}_{200}$ : Displacement at $200 \mathrm{~g}$ pressure

PBA: Proportion of "banana" area

ECM: Extracellular matrix.

\section{Data Availability}

The data used to support the findings of this study are available from the corresponding author upon request.

\section{Conflicts of Interest}

The authors declare that there is no conflict of interests regarding the publication of this paper.

\section{Authors' Contributions}

Haidong Peng, Feng Jin, and Dengpeng Meng contributed equally to this work.

\section{Acknowledgments}

The authors gratefully acknowledge supports from the National Natural Science Foundation of China (NSFC) through the research projects (NO. 81704147) and the technical support of biomechanical test by the Shanghai Science and Technology Committee (STCSM) through the research projects (No. 18401901600).

\section{References}

[1] W. J. Wang, H. Y. Yeung, W. C. W. Chu et al., "Top theories for the etiopathogenesis of adolescent idiopathic scoliosis," Journal of Pediatric Orthopaedics, vol. 31, 1 Suppl, pp. S14S27, 2011.

[2] J.-W. M. Kouwenhoven and R. M. Castelein, "The pathogenesis of adolescent idiopathic Scoliosis," Spine, vol. 33, no. 26, pp. 2898-2908, 2008.

[3] S. Zheng, H. Zhou, B. Gao et al., "Estrogen promotes the onset and development of idiopathic scoliosis via disproportionate endochondral ossification of the anterior and posterior column in a bipedal rat model," Experimental \& Molecular Medicine, vol. 50, no. 11, pp. 1-11, 2018.

[4] R. R. Banala, S. K. Vemuri, M. Penkulinti, G. R. AV, and S. GPV, "Development of novel animal model for studying scoliosis using a noninvasive method and its validation through gene-expression analysis," Asian Spine Journal, vol. 13, no. 1, pp. 126-134, 2019.

[5] A. T. Masi, J. L. Dorsch, and J. Cholewicki, “Are adolescent idiopathic scoliosis and ankylosing spondylitis counter-opposing conditions? A hypothesis on biomechanical contributions predisposing to these spinal disorders," Clinical and Experimental Rheumatology, vol. 21, no. 5, pp. 573-580, 2003.

[6] D. Xie, J. Zhang, W. Ding et al., "Abnormal change of paravertebral muscle in adult degenerative scoliosis and its association with bony structural parameters," European Spine Journal, vol. 28, no. 7, pp. 1626-1637, 2019.

[7] A.-A. K. Yousefi, M. A. Nazari, P. Perrier, M. S. Panahi, and Y. Payan, "A new model of passive muscle tissue integrating collagen fibers: consequences for muscle behavior analysis," Journal of the Mechanical Behavior of Biomedical Materials, vol. 88, pp. 29-40, 2018.
[8] Y. Liu, A. Pan, Y. Hai, W. Li, L. Yin, and R. Guo, “Asymmetric biomechanical characteristics of the paravertebral muscle in adolescent idiopathic scoliosis," Clinical Biomechanics, vol. 65, pp. 81-86, 2019.

[9] C. Wong, K. Gosvig, and S. Sonne-Holm, "The role of the paravertebral muscles in adolescent idiopathic scoliosis evaluated by temporary paralysis," Scoliosis and Spinal Disorders, vol. 12, no. 1, 2017.

[10] R. Mayne and R. D. Sanderson, "The extracellular matrix of skeletal muscle," Collagen \& Related Research, vol. 5, pp. 449-468, 2012.

[11] J. Grasa, M. Sierra, N. Lauzeral, M. J. Muñoz, F. J. MianaMena, and B. Calvo, "Active behavior of abdominal wall muscles: experimental results and numerical model formulation," Journal of the Mechanical Behavior of Biomedical Materials, vol. 61, pp. 444-454, 2016.

[12] J. Gindre, M. Takaza, K. M. Moerman, and C. K. Simms, "A structural model of passive skeletal muscle shows two reinforcement processes in resisting deformation," Journal of the Mechanical Behavior of Biomedical Materials, vol. 22, no. 3, pp. 84-94, 2013.

[13] C. Zetterberg, R. Björk, R. Örtengren, and G. B. J. Andersson, "Electromyography of the paravertebral muscles in idiopathic scoliosis: measurements of amplitude and spectral changes under load," Acta Orthopaedica, vol. 55, no. 3, pp. 304-309, 1984.

[14] A. S. de Oliveira, P. E. S. Gianini, P. M. F. Camarini, and D. Bevilaqua-Grossi, "Electromyographic analysis of paravertebral muscles in patients with idiopathic scoliosis," Spine, vol. 36, no. 5, pp. E334-E339, 2011.

[15] K. Kindsfater, T. Lowe, D. Lawellin, D. Weinstein, and J. Akmakjian, "Levels of platelet calmodulin for the prediction of progression and severity of adolescent idiopathic scoliosis," The Journal of Bone \& Joint Surgery, vol. 76, no. 8, pp. 11861192, 1994.

[16] M. Kjaer, "Role of extracellular matrix in adaptation of tendon and skeletal muscle to mechanical loading," Physiological Reviews, vol. 84, no. 2, pp. 649-698, 2004.

[17] M. Böl, A. E. Ehret, K. Leichsenring, C. Weichert, and R. Kruse, "On the anisotropy of skeletal muscle tissue under compression," Acta Biomaterialia, vol. 10, no. 7, pp. 32253234, 2014.

[18] M. Wajchenberg, D. E. Martins, R. d. P. Luciano et al., "Histochemical analysis of paraspinal rotator muscles from patients with adolescent idiopathic scoliosis: a cross-sectional study," Medicine, vol. 94, no. 8, p. e598, 2015.

[19] F. H. Epstein, W. A. Border, and N. A. Noble, "Transforming growth factor $\beta$ in tissue fibrosis," The New England Journal of Medicine, vol. 331, no. 19, pp. 1286-1292, 1994.

[20] R. Nowak, M. Kwiecien, M. Tkacz, and U. Mazurek, “Transforming growth factor-beta (TGF- $\beta$ ) signaling in paravertebral muscles in juvenile and adolescent idiopathic scoliosis," BioMed Research International, vol. 2014, Article ID 594287, 14 pages, 2014

[21] J. F. Sarwark, K. W. Dabney, S. K. Salzman et al., "Experimental scoliosis in the Rat," Spine, vol. 13, no. 5, pp. 466-471, 1988.

[22] L. Liu, Y. Zhu, X. Han, and Y. Wu, "The creation of scoliosis by scapula-to-contralateral ilium tethering procedure in bipedal rats," Spine, vol. 36, no. 17, pp. 1340-1349, 2011.

[23] M. Ashina, L. Bendtsen, R. Jensen, F. Sakai, and J. Olesen, "Muscle hardness in patients with chronic tension-type 
headache: relation to actual headache state," Pain, vol. 79, no. 2, pp. 201-205, 1999.

[24] Á. Oliva-Pascual-Vaca et al., “Assessment of paraspinal muscle hardness in subjects with a mild single scoliosis curve: a preliminary myotonometer study," Journal of Manipulative and Physiological Therapeutics, vol. 37, no. 5, pp. 326-333, 2015.

[25] J. Ylinen, I. Teittinen, V. Kainulainen, H. Kautiainen, K. Vehmaskoski, and A. Häkkinen, "Repeatability of a computerized muscle tonometer and the effect of tissue thickness on the estimation of muscle tone," Physiological Measurement, vol. 27, no. 9, pp. 787-796, 2006.

[26] B. D. Steinberg, "Evaluation of limb compartments with increased interstitial pressure. An improved noninvasive method for determining quantitative hardness," Journal of Biomechanics, vol. 38, no. 8, pp. 1629-1635, 2005.

[27] M. Amani, M. Rahmati, M. Fathi, and H. Ahmadvand, "Reduce muscle fibrosis through exercise via NRG1/ErbB2 modification in diabetic rats," Journal of Diabetes Research, vol. 2020, Article ID 6053161, 8 pages, 2020.

[28] K. A. Zapata, S. S. Wang-Price, D. J. Sucato, and M. DempseyRobertson, "Ultrasonographic measurements of paraspinal muscle thickness in adolescent idiopathic Scoliosis," Pediatric Physical Therapy, vol. 27, no. 2, pp. 119-125, 2015.

[29] J. Jiang, Y. Meng, X. Jin et al., "Volumetric and fatty infiltration imbalance of deep paravertebral muscles in adolescent idiopathic scoliosis," Medical Science Monitor, vol. 23, pp. 20892095, 2017.

[30] A. F. Mannion, M. Meier, D. Grob, and M. Müntener, "Paraspinal muscle fibre type alterations associated with scoliosis: an old problem revisited with new evidence," European Spine Journal, vol. 7, no. 4, pp. 289-293, 1998.

[31] N. Shafaq, A. Suzuki, A. Matsumura et al., "Asymmetric degeneration of paravertebral muscles in patients with degenerative lumbar scoliosis," Spine, vol. 37, no. 16, pp. 1398-1406, 2012.

[32] C. H. Kang, M. J. Shin, S. M. Kim, S. H. Lee, and C. S. Lee, "MRI of paraspinal muscles in lumbar degenerative kyphosis patients and control patients with chronic low back pain," Clinical Radiology, vol. 62, no. 5, pp. 479-486, 2007.

[33] Q. Li, G.-b. Zhong, Z.-d. Liu, and L.-f. Lao, "Effect of asymmetric tension on biomechanics and metabolism of vertebral epiphyseal plate in a rodent model of scoliosis," Orthopaedic Surgery, vol. 9, no. 3, pp. 311-318, 2017.

[34] R. L. Lieber and J. Fridén, "Functional and clinical significance of skeletal muscle architecture," Muscle \& Nerve, vol. 23, no. 11, pp. 1647-1666, 2000.

[35] B. Hernández, E. Peña, G. Pascual et al., "Mechanical and histological characterization of the abdominal muscle. A previous step to modelling hernia surgery," Journal of the Mechanical Behavior of Biomedical Materials, vol. 4, no. 3, pp. 392-404, 2011.

[36] P. P. Purslow, "Muscle fascia and force transmission," Journal of Bodywork and Movement Therapies, vol. 14, no. 4, pp. 411$417,2010$.

[37] I. H. Koh, H. J. Kang, S. W. Jeon, J. H. Park, and Y. R. Choi, "Passive skeletal muscle excursion after tendon rupture correlates with increased collagen content in muscle," Yonsei Medical Journal, vol. 55, no. 5, pp. 1395-1399, 2014.

[38] M. Mohammadkhah, P. Murphy, and C. K. Simms, "Collagen fibril organization in chicken and porcine skeletal muscle perimysium under applied tension and compression," Journal of the Mechanical Behavior of Biomedical Materials, vol. 77, pp. 734-744, 2018.

[39] F. Trensz, F. Lucien, V. Couture et al., "Increased microenvironment stiffness in damaged myofibers promotes myogenic progenitor cell proliferation," Skeletal Muscle, vol. 5, no. 1, 2015.

[40] R. L. Lieber and S. R. Ward, "Cellular mechanisms of tissue fibrosis. 4. Structural and functional consequences of skeletal muscle fibrosis," American Journal of Physiology. Cell Physiology, vol. 305, no. 3, pp. C241-C252, 2013.

[41] T. Nishimura, K. Ojima, A. Liu, A. Hattori, and K. Takahashi, "Structural changes in the intramuscular connective tissue during development of bovine semitendinosus muscle," Tissue \& Cell, vol. 28, no. 5, pp. 527-536, 1996.

[42] S. H. Fang, T. Nishimura, and K. Takahashi, "Relationship between development of intramuscular connective tissue and toughness of pork during growth of pigs," Journal of Animal Science, vol. 77, no. 1, pp. 120-130, 1999.

[43] W. Friess, "Collagen - biomaterial for drug delivery," European Journal of Pharmaceutics and Biopharmaceutics, vol. 45, no. 2, pp. 113-136, 1998.

[44] I. H. Koh, H. J. Kang, W. T. Oh, J. J. Hong, and Y. R. Choi, "Correlation between change in muscle excursion and collagen content after tendon rupture and delayed repair," Journal of Orthopaedic Surgery and Research, vol. 12, no. 1, p. 15, 2017.

[45] R. J. McCormick, "The flexibility of the collagen compartment of muscle," Meat Science, vol. 36, no. 1-2, pp. 79-91, 1994.

[46] M. A. A. Mahdy, "Skeletal muscle fibrosis: an overview," Cell and Tissue Research, vol. 375, no. 3, pp. 575-588, 2019.

[47] M. Lodyga and B. Hinz, "TGF- $\beta 1$ - A truly transforming growth factor in fibrosis and immunity," Seminars in Cell \& Developmental Biology, vol. 101, pp. 123-139, 2020.

[48] I. I. Ryzhkov, E. E. Borzilov, M. I. Churnosov, A. V. Ataman, A. A. Dedkov, and A. V. Polonikov, "Transforming growth factor beta 1 is a novel susceptibility gene for adolescent idiopathic scoliosis," Spine, vol. 38, no. 12, pp. e699-e704, 2013.

[49] H. Xu, G. Qiu, Z. Wu et al., "Expression of transforming growth factor and basic fibroblast growth factor and core protein of proteoglycan in human vertebral cartilaginous endplate of adolescent idiopathic scoliosis," Spine, vol. 30, no. 17, pp. 1973-1978, 2005.

[50] S. J. Moravec and J. F. Cleall, "An assessment of posture in bipedal rats," The American Journal of Anatomy, vol. 180, no. 4, pp. 357-364, 1987.

[51] A. S. Bailey, F. Adler, S. Min Lai, and M. A. Asher, "A comparison between bipedal and quadrupedal rats," Spine, vol. 26, no. 14, pp. E308-E313, 2001. 